\title{
Space-Frequency Balance in Biorthogonal Wavelets
}

\author{
D. M. Monro and B. G Sherlock \\ School of Electronic and Electrical Engineering, University of Bath \\ Claverton Down, BA2 7AY, England \\ e-mail: D.M.Monro@bath.ac.uk \\ B.G.Sherlock@bath.ac.uk \\ Fax: +44 1225826073 \\ Internet: http://dmsun4.bath.ac.uk/
}

\begin{abstract}
This paper shows how to design good biorthogonal FIR filters for wavelet image compression by balancing the space and frequency dispersions of analysis and synthesis lowpass filters. A quality metric is proposed which can be computed directly from the filter coefficients. By optimizing over the space of FIR filter coefficients, a filter bank can be found which minimizes the metric in about 60 seconds on a high performance workstation. The metric contains three parameters which weight the space and frequency dispersions of the low pass analysis and synthesis filters. A series of biorthogonal, symmetric wavelet filters of length 10 was found, each optimized for different weightings. Each of these filter banks was then evaluated by compressing and decompressing five test images at three compression ratios. Selecting each optimum provides fifteen sets of parameters corresponding to filter banks which maximize the PSNR in each case. The average of these parameters was used to define a 'mean' filter bank, which was then evaluated on the test images. Individual images can produce substantially different weightings of the time dispersion at the optimum, but the PSNR of the mean filter is normally close to the optimum. The mean filter also compares favourably with a maximum regularity biorthogonal filter of the same length.
\end{abstract}

\section{BACKGROUND}

The theory of continuous and discrete wavelet transforms [1, 2] has inspired much basic and applied research in signal and image processing, as well as revitalizing the study of sub-band filtering [3, 4, 5]. The Discrete Wavelet Transform (DWT) is obtained by repeated filtering and sub-sampling into two bands with low- and high-pass Finite Impulse Response (FIR) filters called the analysis filters. The inverse process makes use of the synthesis FIR filters, and gives perfect reconstruction if the wavelet is biorthogonal. This is easily shown to be the case [4] if the lowpass analysis filter coefficients $\left\{c_{0}, \ldots, c_{L-1}\right\}$ and synthesis filter coefficients $\left\{u_{0}, \ldots, u_{L-1}\right\}$ satisfy $\sum_{n} c_{n} u_{n+2 j}=\delta_{j, 0}$. Wavelets exist only if zero order regularity conditions are satisfied,

$$
\sum_{n} c_{n}=\sqrt{2}, \quad \sum_{n}(-1)^{n} u_{n}=0, \text { and } \quad \sum_{n}(-1)^{n} c_{n}=0 .
$$

The lower limit on the time-frequency resolution that can be obtained with wavelet transforms is given theoretically by the Heisenberg uncertainty relation [6] which, applied to the signal $f(t)$, is expressed by the inequality:

$$
\Delta \omega \Delta t \geq 1 / 2
$$

where

$$
\begin{aligned}
& \Delta t^{2}=\frac{\int \tau^{2} f(\tau) d \tau}{\int f(\tau)^{2} d \tau} \text { measures the pulse width, } \\
& \tau=t-\bar{t}, \text { and } \\
& \Delta \omega^{2}=\frac{\int \omega^{2}|F(\omega)|^{2} d \omega}{\int|F(\omega)|^{2} d \omega} \text { is the bandwidth, }
\end{aligned}
$$

and $F(\omega)$ is the Fourier transform of $f(t)$.

The uncertainty inequality quantifies the fact that the time and frequency localization of information cannot simultaneously be arbitrarily small. It can be shown that the continuous, infinite time, Gabor wavelet [6] is the only wavelet which achieves this minimum. The lower the Heisenberg uncertainty, the better is the resolving power of the wavelet. The uncertainty is invariant over the scales used in the DWT; as data is filtered and subsampled, the time resolution is successively halved and the frequency resolution is doubled, preserving their product $\Delta \omega \Delta t$.

We can compute the bandwidth $\Delta \omega$ and time dispersion $\Delta t$ directly for a FIR filter from its coefficients $\left\{h_{i}\right\}$ :

$$
\Delta \omega^{2}=\frac{\pi^{2}}{3}+4 \sum_{n=0}^{L-2} \sum_{m=n+1}^{L-1} \frac{(-1)^{m-n}}{P(m-n)^{2}} h_{m} h_{n}
$$




$$
\begin{aligned}
& \text { where } \quad P=\sum_{n=0}^{L-1} h_{n}^{2} \\
& \Delta t^{2}=\frac{1}{P} \sum_{n=0}^{L-1}(n-\bar{t})^{2} h_{n}^{2} \\
& \text { where } \\
& \qquad \bar{t}=\frac{\sum_{n} n h_{n}}{\sum_{n} h_{n}} .
\end{aligned}
$$

\section{A BALANCED UNCERTAINTY METRIC}

The Heisenberg uncertainty product $\Delta \omega \Delta t$ is not itself a useful figure of merit for wavelet design. In our earlier work [7] we introduced an uncertainty metric for the orthonormal case, which forms a weighted balance between $\Delta \omega$ and $\Delta t$. We showed that orthonormal wavelets giving good MSE and visual quality could be designed by appropriate trade-off between frequency and time dispersion. The results were superior to those of maximum regularity wavelets, both in MSE and organized visual trials.

We now propose the following balanced uncertainty metric for biorthogonal wavelets:

$$
M\left(k_{2}, k_{3}, k_{4}\right)=\Delta \omega^{2}{ }_{a}+k_{2} \Delta t^{2}{ }_{a}+k_{3} \Delta \omega^{2}{ }_{s}+k_{4} \Delta t^{2}{ }_{s}
$$

where subscripts $a$ and $s$ refer to the analysis and synthesis filters respectively, and the parameters $k_{2}, k_{3}$ and $k_{4}$ specify the relative importance attached to the time and frequency resolutions. To design a wavelet for any desired balance, we choose values for $k_{2}, k_{3}$ and $k_{4}$, and minimize $M\left(k_{2}, k_{3}, k_{4}\right)$ directly from the FIR filter coefficients using equations 1 and 2 . This enables us to search for good wavelets without the cost of repeated compression and decompression cycles.

\begin{tabular}{|c|c|c|}
\hline$i$ & Analysis $c_{i}$ & Synthesis $u_{i}$ \\
\hline 0,1 & 0.714164424 & 0.675200974 \\
\hline$-1,2$ & 0.070679818 & 0.120013751 \\
\hline$-2,3$ & -0.105948358 & -0.08794428 \\
\hline$-3,4$ & 0.016675933 & -0.00053087 \\
\hline$-4,5$ & 0.011534965 & 0.000367208 \\
\hline
\end{tabular}

Table 2. Filter coefficients for the 'mean' wavelet.

\section{DESIGN OF BIORTHOGONAL BALANCED UNCERTAINTY WAVELETS}

Wavelets were designed for 512 different uncertainty balances by allowing $k_{2}, k_{3}$ and $k_{4}$ each to assume eight discrete values and minimizing $M\left(k_{2}, k_{3}, k_{4}\right)$ for each combination of parameter values. Minimization was done by simulated annealing [8] over the FIR filter coefficients. The analysis and synthesis filters were both symmetric with

$$
\text { Boats Luminance Compressed 20:1 (0.4bpp), K3 }=1.32
$$

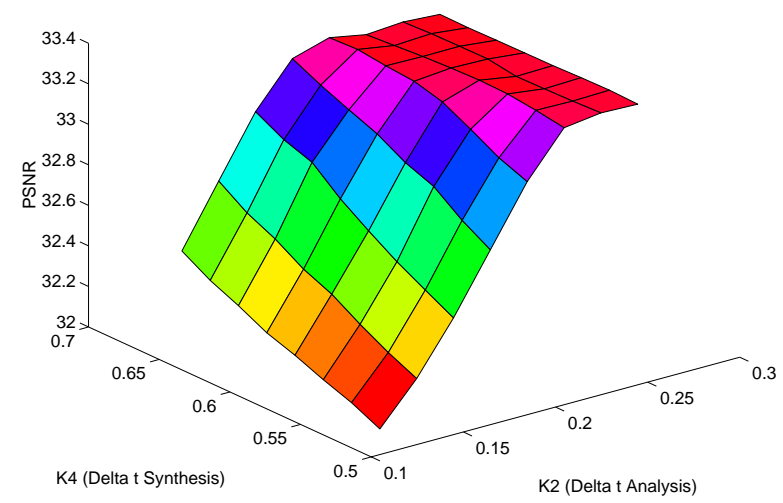

Figure 1. The variation of the MSE with the space dispersion parameters $k_{2}$ and $k_{4}$, on the Boats image with $k_{3}=1.32$.

\begin{tabular}{|c|c|c|c|c|c||}
\hline Wavelet & Gold Hill & Barbara 1 & Barbara 2 & Boats & Lenna \\
\hline Optimum for image & 31.85 & 28.70 & 28.29 & 33.32 & 33.84 \\
\hline 'Mean' & 31.69 & 28.41 & 28.24 & 33.04 & 33.80 \\
\hline Maximum Regularity & 31.44 & 27.68 & 27.35 & 33.26 & 33.76 \\
\hline Optimum for Boats image & 31.53 & 27.72 & 27.43 & 33.32 & 33.72 \\
\hline Optimum for Lenna image & 31.66 & 28.31 & 28.19 & 33.02 & 33.84 \\
\hline
\end{tabular}

Table 1. PSNR performance of the 'mean' wavelet at $20: 1$ compression $(0.4 \mathrm{bpp})$ compared with the individual optimum wavelets, and the maximum regularity wavelet of the same length. 


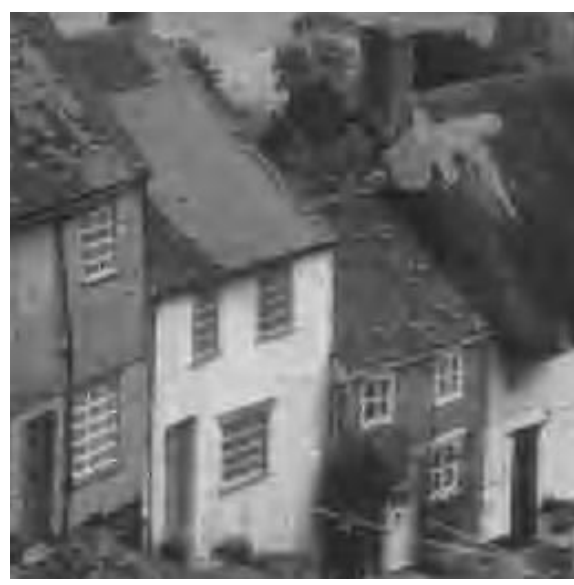

(a) Optimum wavelet for Gold Hill, PSNR= 29.52

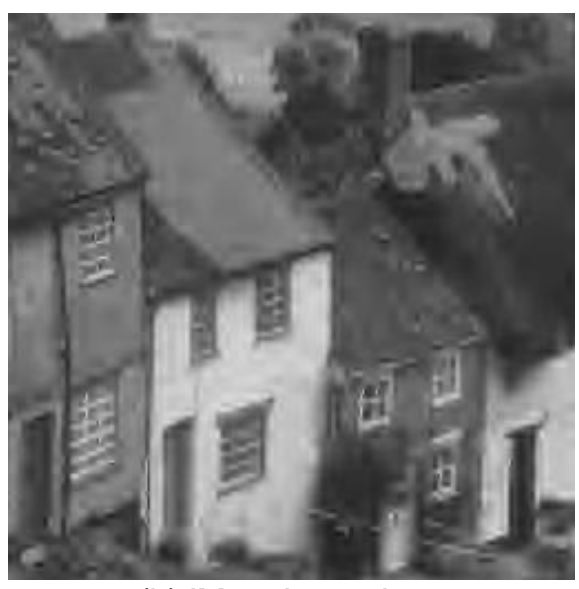

(b) 'Mean' wavelet, PSNR $=29.49$

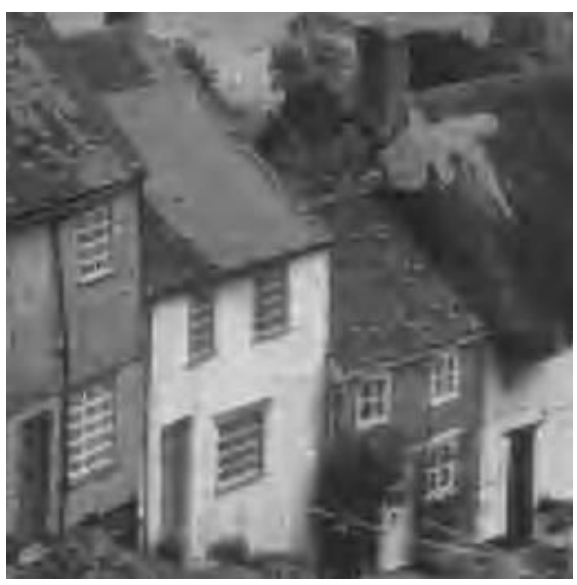

(c) Maximum regularity, PSNR $=29.46$

Figure 2. Results at 40:1 (0.2bpp) for a fragment of 'Gold Hill' using 10th order biorthogonal symmetric wavelets.

10 taps. Each minimization took approximately 60 seconds on a Sun Ultra 170 workstation.

Using our implementation of Shapiro's zerotree coder [9], each of the 512 wavelets was used to compress the five test images listed in Table 1 at compressions of 20:1, 40:1 and 80:1, and the resultant errors were recorded.

As an example, Figure 1 shows the variation of the PSNR error as a function of the space dispersion parameters $k_{2}$ and $k_{4}$, for fixed $k_{3}$ for the boats image at 20:1.

The parameter values yielding lowest error for each of the 15 test image / compression ratio combinations were obtained. A compromise wavelet intended to give good performance over all test images was obtained by averaging the above 15 sets of $k$-parameter values, to get $k_{2}=0.61$, $k_{3}=1.33$, and $k_{4}=0.55$. The wavelet coefficients found by optimizing $M\left(k_{2}, k_{3}, k_{4}\right)$ are given in Table 2.

Additionally, a maximum regularity $10 / 10$ wavelet (wavelet D of [10]) was used to compress each of the 5 test images at the three compression ratios. It was found that the best of the 512 balanced uncertainty wavelets gave lower error than the maximum regularity wavelet in every case. The 'mean' wavelet improved over maximum regularity in 12 out of 15 cases. Table 1 compares the PSNR performance of the 'mean' wavelet at 40:1 compression with the individual optimum wavelets, and the maximum regularity wavelet of the same length. Also included in the table are the best wavelets for Boats and Lenna applied across all five images.

\section{APPLICATION TO FINGERPRINTS}

The use of wavelets for compressing fingerprint images for use in Automated Fingerprint Identification Systems (AFIS) is of considerable commercial importance because the US Federal Bureau of Investigation (FBI) has specified the use of a wavelet technique for fingerprint image compression, as well as a particular wavelet for use within this technique. The FBI wavelet is a maximum regularity spline wavelet of length 9/7 [11].

In our earlier work [12], we searched the space of biorthogonal wavelets to find wavelets which are optimal for the compression of fingerprint images. This work produced a length $10 / 10$ wavelet, called S10, which minimized the rms error between original and reconstructed fingerprint images at 20:1 compression.

We compressed fingerprint images using the 'mean' wavelet, the FBI wavelet and the S10 wavelet. Table 3 shows typical rms errors as well as a psychovisual ranking of the reconstructed images by two police fingerprint experts. Figure 3 shows detail from one of the three fingerprints used in the trial.

\begin{tabular}{|c|c|c||}
\hline Wavelet & $\begin{array}{c}\text { rms error } \\
\text { at } 20: 1\end{array}$ & $\begin{array}{c}\text { psychovisual } \\
\text { ranking }\end{array}$ \\
\hline 'Mean' & 7.26 & 1 (best) \\
\hline S10 & 6.88 & 2 \\
\hline FBI & 7.20 & 3 (worst) \\
\hline \multicolumn{2}{|c|}{ Table 3. Wavelets compared on fingerprints } \\
\hline
\end{tabular}

Table 3. Wavelets compared on fingerprints

The fact that the 'mean' wavelet achieves the best psychovisual results is remarkable, because the other two wavelets were specifically designed for optimal performance on fingerprint images. This result illustrates the validity of the balanced uncertainty metric, and vindicates the use of uncertainty as a powerful tool for the design of wavelets.

\section{DISCUSSION}

Previous work showed that significant PSNR and visual improvement over maximum regularity wavelets was obtained by selecting orthonormal wavelets for the balance of their time and frequency dispersions [7]. Here we have 


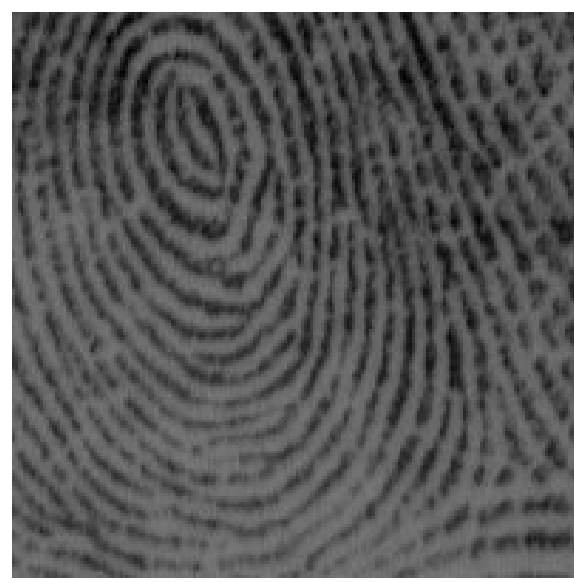

(a) Original

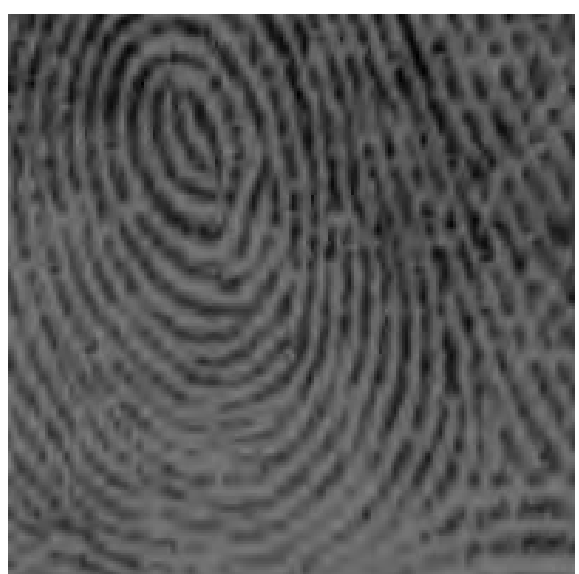

(b) Compressed by FBI wavelet

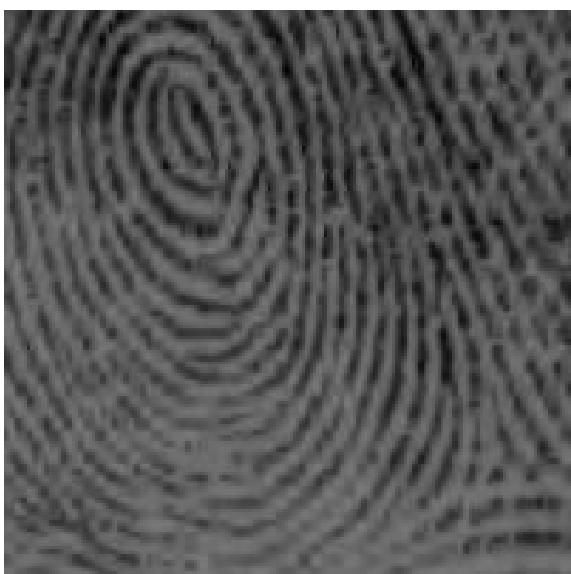

(c) Compressed by 'mean' wavelet

Figure 3. Compression results at 20:1 (0.4bpp) for a 200x200 pixel fragment of a typical fingerprint.

extended this result to biorthogonal wavelets. The space-frequency characteristics of wavelet filters are often held to be an important attribute [5], but in practice consideration of these characteristics in designing wavelets is largely ignored. We use a metric which balances scale and frequency to design wavelet filters, and examine the PSNR of the filter banks in image compression using a specific family of wavelets on several test images. The results show this to be a good approach.

\section{CONCLUSIONS}

We have introduced a metric based on balanced uncertainty for the design of biorthogonal wavelets. Minimization of the metric balances the time and frequency spreads of the analysis and synthesis filter responses. Because the metric can be computed directly from the filter coefficients, it can be used to optimize wavelets for image compression without the cost of repeatedly compressing and decompressing images.

Perhaps the most important comparison is with the 'maximum regularity' approach, which tends to give the filter a flat response and sharp cutoff in the frequency domain, at the expense of ringing. Our results show this to be a suboptimum design principle in selecting wavelets for image compression. With suitably chosen parameters, the use of balanced uncertainty delivers significantly higher PSNR than is obtained with a wavelet chosen for maximum regularity.

Since the 'mean' wavelet performs considerably better than a wavelet optimised for the Boats or Lenna images (see Table 1), an important conclusion is that wavelets should not be designed on the basis of their performance on a single image. Several of the trials using Lenna produced $k_{2}, k_{3}, k_{4}$ parameters near the extremes of their ranges. Despite its popularity, if a single test image is to be used for designing and comparing wavelets for compression, we suggest it should not be this one.

\section{REFERENCES}

[1] Daubechies, I., 'Orthonormal bases of compactly supported wavelets', Comm. Pure Appl. Math., Vol. 41, pp 909-966, 1988.

[2] Mallat, S., 'A theory for multiresolution signal decomposition: The wavelet representation', IEEE Trans. Pattern Anal. Mach. Intel., Vol. 11, July 1989.

[3] Smith, M. J. T. and Barnwell, T. P., 'A new filter bank theory for time-frequency representation', IEEE Trans. Acoust., Speech and Signal Process., Vol ASSP-35, No. 3, pp 314-327, March 1987. [4] Antonini, M., Mathieu, P., and Daubechies, I., 'Image coding using wavelet transform', IEEE Trans. Image Process., Vol.1, No. 2, pp 205-220, April 1992.

[5] Vetterli, M., and Herley, C., 'Wavelets and filter banks: Theory and design', IEEE Trans. Signal Process., Vol. 40, No. 9, pp. 2207-2232, September 1992.

[6] Gabor, D. 'Theory of Communication', J. of the IEE, Vol. 93, pp. 429-457, 1946.

[7] Monro, D. M., Bassil, B. E., and Dickson, G. J., 'Orthonormal wavelets with balanced uncertainty', IEEE Int. Conf. Image Process. (ICIP '96), Lausanne, Switzerland, September 1996.

[8] Kirkpatrick, S., Gelatt, C. D., and Vecchi, M. P., 'Optimization by simulated annealing', Science, Vol. 220, pp. 671-679, 1983.

[9] Shapiro, J. M., 'Embedded image coding using zerotrees of wavelet coefficients', IEEE Trans. Signal Process., Vol. 41, No. 12, pp. 3445-3462, December 1993.

[10] Da Silva, E. A. B., and Ghanbari, M.,'On the performance of linear phase wavelet transforms in low bit-rate image coding', IEEE Trans. Image Process., Vol. 5, No. 5, pp. 689-704, May 1996.

[11] FBI Document IAFIS-IC-0110V2, 'WSQ gray-scale fingerprint image compression specification', February 1993.

[12] B. G. Sherlock and D. M. Monro, 'Optimized Wavelets for Fingerprint Compression', IEEE Int. Conf. Acoust., Speech Signal Process. (ICASSP-96), Atlanta, Georgia,Vol. III, pp. 1447-1450, May 1996. 